\title{
The relationship between intelligence and orientation discrimination skills
}

\author{
Kyriaki Mikellidou $^{1,2}$, Nefeli Lambrou ${ }^{2,3}$, Ellada Georgiou ${ }^{1} \&$ Marios Avraamides $^{1,2,4}$
}

${ }^{1}$ Department of Psychology and Center for Applied Neuroscience, University of Cyprus, Nicosia, Cyprus; ${ }^{2}$ Centre of Applied Neuroscience, Nicosia, Cyprus; ${ }^{3}$ Department of Psychology, University of Glasgow, Scotland, United Kingdom; ${ }^{4}$ CYENS Research Centre, Nicosia, Cyprus

\section{Abstract}

We investigate the notion that basic visual information is acting as a building block for more complex cognitive processes. Specifically, we measure individual orientation discrimination thresholds report good correlations against IQ, verbal-IQ and non-verbal IQ scores. Further we calculate correlations between the four WASI-II subtests and orientation discrimination thresholds, with Matrix Reasoning maintaining the strongest relationship, even when controlling for the effects of the other three subtests. Vocabulary raw scores which quantify the ability to describe verbally a specific word, showed the second strongest correlation, when controlling for the effects of Matrix Reasoning and Block Design, but not Similarities. Our results demonstrate that low-level visual abilities and high-level cognitive processes are more tightly interwoven together than previously thought, generating evidence to show that vision is tightly linked to human cognition.

\section{Statement of Relevance}

Vision is one of the building blocks of our world experience through thoughts, emotions and actions. It is undeniably the sense upon which we rely the most, while working, driving, reading a book, dancing. So, it should come as no surprise that the intelligence quotient (IQ), widely used in the modern world to assess the cognitive abilities of a person in relation to their age group correlates well with visual abilities. Previous studies have demonstrated that pitch and colour discrimination, motion discrimination and contrast discrimination abilities are tightly linked to IQ. In this study we demonstrate for the first time, that the simplest visual ability, orientation discrimination which is computed by neurons in the primary visual cortex is tightly linked to intelligence. We believe a simple and quick orientation discrimination task could be used as an index of intelligence in clinical populations and other species, in addition to traditional measures. 


\section{Introduction}

Past research has documented a link between intelligence and low-level visual perception, with higher Intelligence Quotient (IQ) associated with faster and more accurate perceptual judgements. This has been demonstrated with a variety of sensory discrimination tasks, including pitch and colour discrimination (Acton \& Schroeder, 2001), motion discrimination (Melnick et al. 2013), and contrast discrimination (Cook et al. 2016; ArranzParaíso \& Serrano-Pedraza, 2018).

In one study, Melnick et al. (2013) asked participants to identify the motion direction of briefly presented gratings of two sizes (small or large). The threshold difference between the two sizes was defined as the "suppression index" (SI) and represented the degree of impairment in motion perception with increasing stimulus size. Previously, Tadin et al. (2003) had shown that the motion direction of high-contrast patterns becomes harder to perceive with increasing stimulus size. This effect was termed "spatial suppression" and is considered a signature of inhibitory mechanisms that make motion-selective neurons less responsive to large, background-like motion patterns. Across two experiments, Melnick et al., reported strong correlations $\left(\mathrm{r}=0.64 ; \mathrm{p}=0.02\right.$ and $\mathrm{r}=0.71 ; \mathrm{p}=10^{-9}$ respectively, 95\% CI) between IQ and SI, supporting the notion that accuracy and speed processing of low-level visual properties predicts intelligence. This result is in line with Galton's original proposition which he failed to support experimentally, that intelligence and simple sensory discriminations operate via common neural processes (Deary, 1994). In support of this idea, Rademaker et al. (2019) showed that population-level response patterns in early visual cortex (specialised in visual processing), represent the contents of working memory alongside new sensory inputs, as when participants were distracted, disruptions of mnemonic information in early visual cortex and decrements in behavioural recall were both observed.

As Tadin et al. (2015) argued, rapid processing of relevant information and suppression of redundant and less informative signals are crucial properties of any system operating on information that exceeds its processing capacity. Tadin et al., replicated Melnick et al.'s (2013) strong link between SI and IQ $(r=0.71)$, showing that wheareas participants with lower IQ scores performed about equally for small and large stimuli (thus a small SI), those with high IQ scores exhibited a large difference in performance between the two sizes. This ties into a broader scheme whereby information suppression in any brain system that operates on 
exceeding capacity, such as working memory, is crucial for its efficiency and accuracy in both sensory discrimination and higher order reasoning.

Perceptual tasks typically correlate well with IQ (usually between 0.2 and 0.4 ), but spatial suppression in particular appears to have a stronger association with IQ scores (correlation $\sim 0.7$ ) as reported by Melnick et al. (2013) and later by Tadin et al. (2014). A question that arises from this past research is whether the tight coupling observed between IQ and visual motion suppression mechanisms, which are thought to reflect the receptive field properties of centre-surround neurons in motion-sensitive area MT, can be also observed with a simple orientation discrimination task. Orientation discrimination skills are determined primarily by V1, which has specialised orientation-tuned neurons (Hubel \& Wiesel, 1968) and is the first brain area that receives visual input from the retina of the eye. Therefore, an orientation discrimination task allows to evaluate the simplest possible visual mechanism that could be driving the visual skills that are commonly tested in intelligence tests.

Thus, the aim of the present study is to determine whether performance on a simple orientation discrimination task can predict intelligence. In the study, orientation discrimination thresholds were measured as a function of the Just Noticeable Difference in degrees, i.e. how much difference in orientation (measured in degrees) is needed for a participant to realise that two orientation stimuli are different. Intelligence was measured using the Wechsler Adult Intelligence Scale and the Wechsler Abbreviated Scale of Intelligence Second Edition (WASIII) test battery comprising Verbal Reasoning, Block Design, Similarities and Matrix Reasoning subtests. To control for the effects of age and education which play a fundamental role in IQ scores, our sample comprised young adults which possessed university-level education. 


\section{Methods}

Two experiments were conducted in which participants were tested with the same orientation discrimination task. However, in Experiment 1 participants' IQ was measured with an online version of the Wechsler Adult Intelligence Scale (WAIS) whereas in Experiment 2 with the Standardized Wechsler Abbreviated Scale of Intelligence (WASI-II). We present the results of the two experiments together.

\section{Participants}

Experiment 1. Twenty-two participants (12 female; age range 18-34) participated in Experiment 1. They all had normal or corrected to normal vision, no neurological disorders and no learning disabilities. They were recruited from the Psychology participant pool of the University of Cyprus, via the internet, and by word of mouth. All participants had universitylevel education. Participants were native Greek speakers but all had excellent knowledge of the English language, as most of them had attended private English schools. Participants received either a monetary reimbursement of $€ 10$ /hour or course credit in exchange for their participation.

Experiment 2. Fifty-nine participants (30 female; age range 18-36) participated in Experiment 2. Inclusion criteria involved normal or corrected to normal vision, no neurological disorders, no learning disabilities and currently in or already possessing university-level education. In total, 10 participants were excluded from the analyses; three had vision that wasn't normal or corrected to normal and failed to inform the experimenter prior to the beginning of the experiment (AM, GT, CGa). Three participants were excluded because no psychometric function could be fitted to their data (CC, RT, IK), Furthermore, one participant was excluded due to photosensitivity with their eyes becoming watery during the psychophysics session and they could not see the stimuli very well (AMi), one participant was an outlier (AS, JND=25), and two participants were excluded (CG \& APat) because they did not complete all parts of the experiment. Therefore, the final analysis included data from 48 participants ( 23 female).

\section{Materials}

\section{Orientation Discrimination Task}

In order to measure orientation discrimination participants were asked to fixate their gaze on a small central fixation square for the whole duration of the experiment and compare the orientation of two sequentially-presented Gabor patches (diameter $=5$ degrees) at an 
eccentricity of 7.5 degrees. The method of constant stimuli was used with the standard stimulus oriented at $45^{\circ}$ and the variable stimulus in one of the following orientations: $30^{\circ}, 33^{\circ}, 36^{\circ}$, $39^{\circ}, 42^{\circ}, 45^{\circ}, 48^{\circ}, 51^{\circ}, 54^{\circ}, 57^{\circ}$, and $60^{\circ}$. After the presentation of the stimuli, participants were asked which one of two sequentially presented Gabor patches was oriented further clockwise. Stimuli were presented on a calibrated 23-inch LCD monitor subtending $26^{\circ}$ (horizontal) by $14.5^{\circ}$. Stimuli were generated with MATLAB (the MathWorks, Natick, MA) using routines from the Psychtoolbox (Brainard, 1997). Responses were collected via a standard keyboard connected via USB to a PC yielding a temporal resolution of 4 ms.

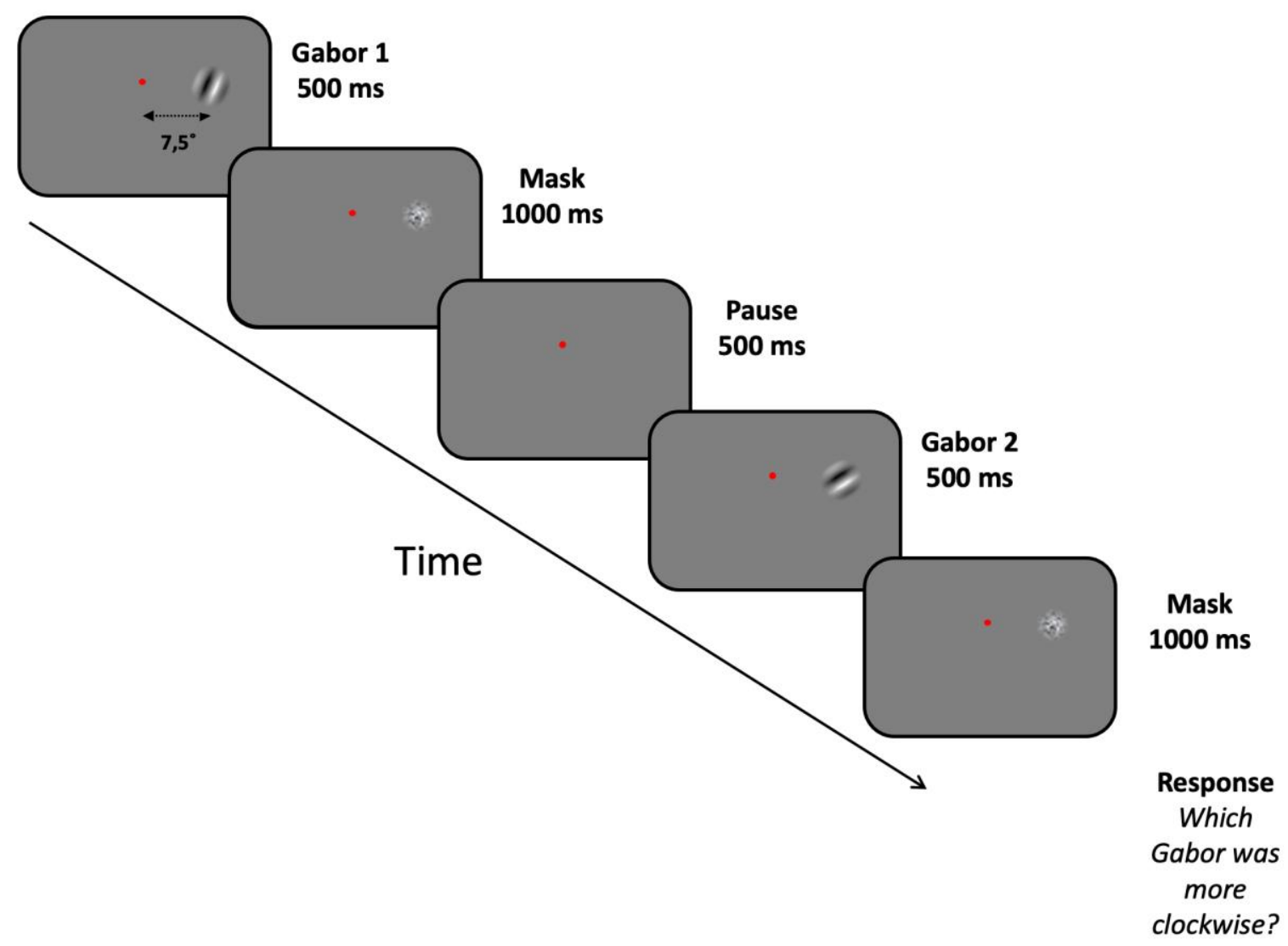

Figure 1

Timeline and stimuli of the two-AFC orientation discrimination task.

\section{Wechsler Adult Intelligence Scale (WAIS) IQ online test}

Two WAIS tests were administered. First, a short questionnaire comprised of 25 questions was administered for 6 minutes to familiarise participants with the pace and type of questions 
(https://wechsleriqtest.com/short-iq-test/). Then, a longer version that included 50 questions was administered for 12 minutes (https://wechsleriqtest.com/50-question-iq-test/).

\section{Standardized Wechsler Abbreviated Scale of Intelligence (WASI-II) battery of tests Participants}

WASI-II measures the intellectual ability of people aged between 6-89 years old. The standardized scaled scores of WASI-II range between 50-160. We used a standardised WASIII for the Greek-Cypriot population in Cyprus in Greek (Spanoudis \& Tourva, 2012), which was the native language our participants (for a detailed description of the four subtests please see Appendix I). To administer the WASI-II we used an answer paper, the book of stimuli, a set of 9 cubes, and a chronometer as required. The four subscales were administered in a fixed sequence that alternates between verbal and non-verbal subscales: (A) Vocabulary participants' ability to describe verbally a specific word was evaluated (B) Block Design participants were asked to recreate a pattern using bi-coloured cubes under a time limit showed the weakest correlation $(C)$ Similarities - evaluation of participants' ability to describe verbally how two words are related (e.g., "how are strawberries and pears similar?"), (D) Matrix Reasoning - participants were asked to indicate the missing piece of a pattern amongst five options. Average time needed for the WASI-II to be completed is 30-45 minutes.

\section{Procedure}

For both experiments, participants were asked to come into the lab well-rested. Upon arriving at the lab they were asked to read an information sheet about the experiment and then to sign an informed consent form. In Experiment 1, participants were first asked to take the online IQ test in English, starting with the short version and then continuing with the long version after, a short break. In Experiment 2, participants completed instead the Standardized WASI-II IQ test in Greek. Participants were seated behind a desk and completed the four subtests that entailed describing words, constructing patterns using wooden cubes, finding similarities between two words, and indicating which picture matched a given pattern.

After completion of the IQ tests, participants performed a two-alternative forced choice (2-AFC) orientation judgement task to measure individual sensitivity in an orientation discrimination task. Participants performed three runs (55 trials each run) of the orientation discrimination task. The first run was considered practice and was not used in the analysis. 


\section{Results}

In Experiment 1, for each participant, 110 trials were used to compute psychometric functions which were fitted with cumulative Gaussians. From this, the Just Noticeable Difference (JND) was calculated in degrees and was plotted as a function of the WAIS-IQ scores. Figure 2 show a strong negative correlation between IQ scores and JND ( $R=0.58$, $\mathrm{p}=0.0049$ ), indicating that participants with a higher intelligence quotient have also more precise orientation discrimination skills. To replicate our results, we used a standardized WASI-II test battery in Greek. This had two benefits. First, it allowed us to confirm the validity of our results by testing participants in their mother tongue. Second, it enabled us to calculate verbal and non-verbal indices of intelligence separately, using the Vocabulary and Similarities subtests for the former and Block Design and Matrix Reasoning for the latter.

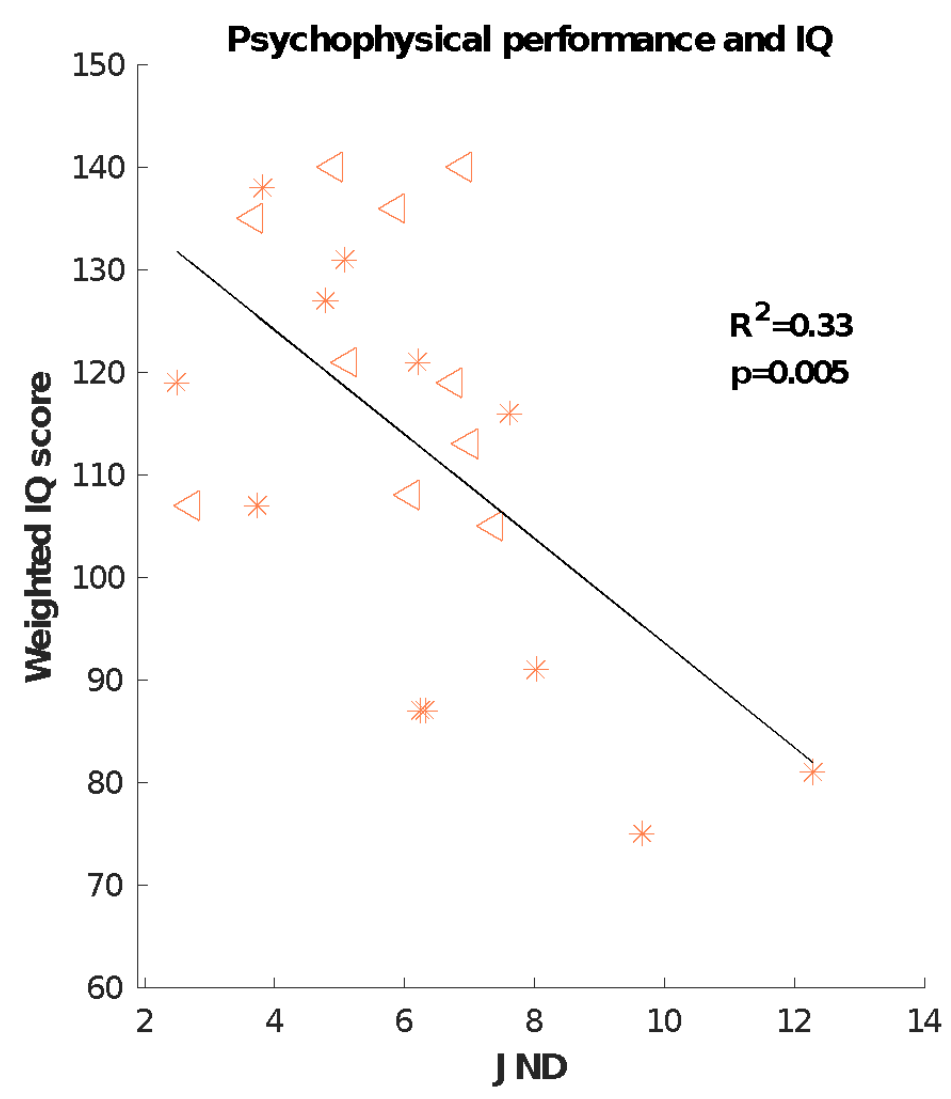

Figure 2

Intelligence Quotient (IQ) as measured by WAIS plotted against Just Noticeable Difference $(J N D)$ in degrees of orientation $(N=22)$. The linear regression model shows a negative 
correlation between the two variables and can explain $33 \%$ of the variance $(p=0.0049)$. Triangles indicate males, stars indicate females.

Replicating the results of Experiment 1, as shown in Figure 3A, we found a negative correlation between Total scaled IQ scores and JND as measured in a 2-AFC orientation judgement task $\left(\mathrm{R}=0.47, \mathrm{R}^{2}=0.23, \mathrm{p}=0.001\right)$. As shown in Figures $3 \mathrm{~b}$ and $3 \mathrm{c}$, we also found negative correlations between scaled Verbal IQ scores and JND $\left(R=0.38, R^{2}=0.14, p=0.008\right)$ and between scaled Non-Verbal IQ scores and JND $\left(\mathrm{R}=0.44, \mathrm{R}^{2}=0.19, \mathrm{p}=0.002\right)$.
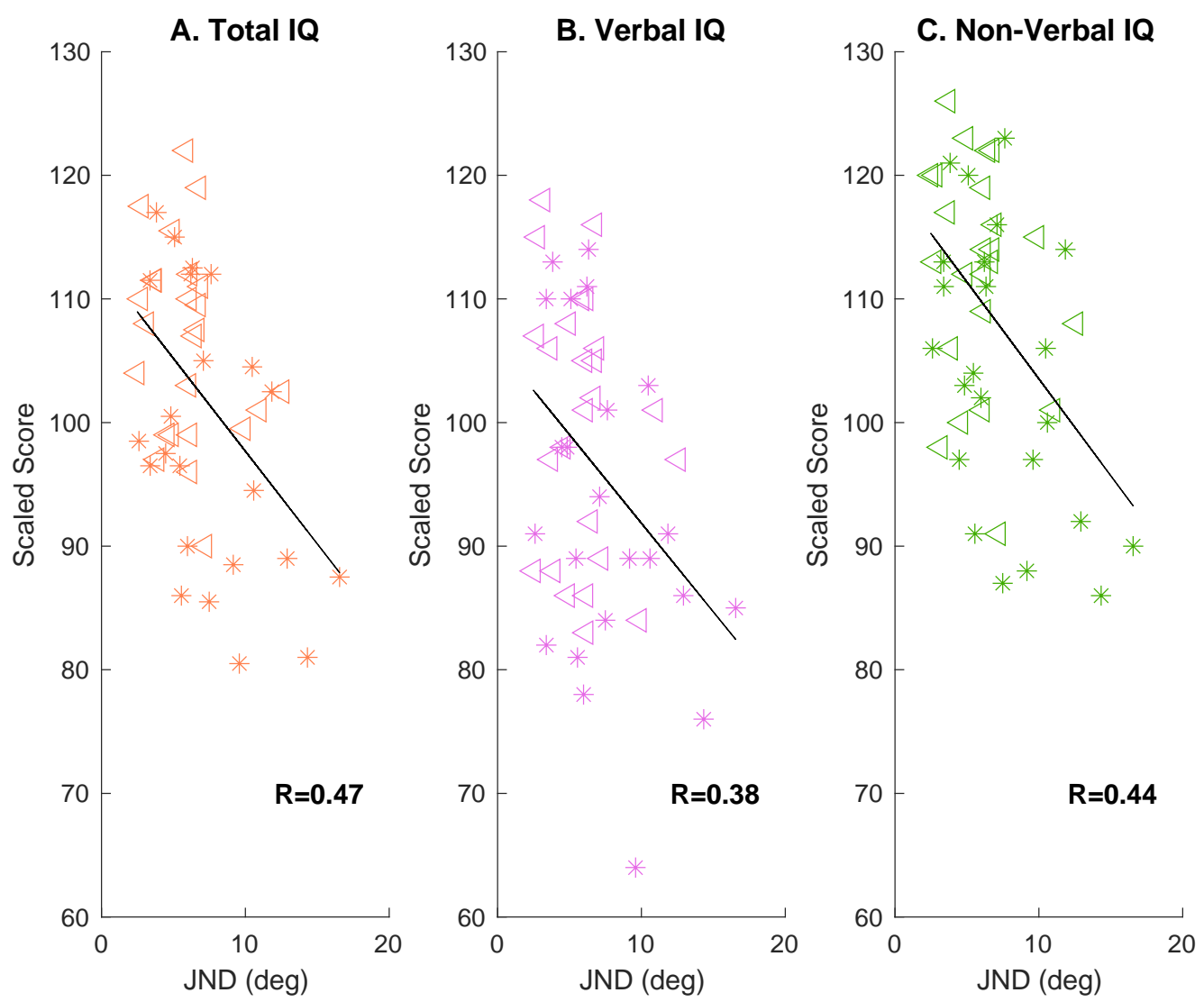

Figure 3

Individual scaled scores for Total, Verbal and Non-verbal IQ correlated against JND in degrees of orientation $(N=48)$. Triangles indicate males, stars indicate females. A. Total Intelligence Quotient (Total IQ) as measured by WASI-II plotted against Just Noticeable Difference $(J N D)$ in degrees of orientation $(N=48)$. The linear regression model shows a 
negative correlation between the two variables $\left(R^{2}=0.22, p=0.001\right)$. B. Scaled Verbal IQ is independently and negatively correlated with JND $\left(R^{2}=0.14, p=0.008\right)$. C. Scaled non-verbal $I Q$ has an independent and even stronger negative correlation with $J N D\left(R^{2}=0.19, p=0.002\right)$.

Subsequently, we correlate each one of the four subtests (Vocabulary, Block Design, Similarities, Matrix Reasoning) with individual JND values. As shown in Figure 4A, Vocabulary raw scores are negatively correlated with JND $\left(R=0.39, R^{2}=0.15, p=0.006\right)$ suggesting that participants with an enriched vocabulary also have more accurate orientation discrimination skills (i.e. smaller JND values). There is also a negative, but weaker correlation between Similarities raw scores and JND shown in Figure $4 C\left(R=0.31, R^{2}=0.1, p=0.032\right)$.

A. Vocabulary

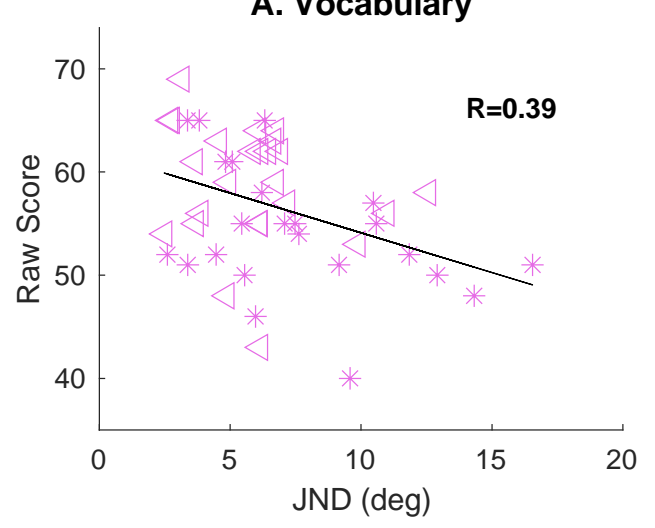

B. Block Design



C. Similarities

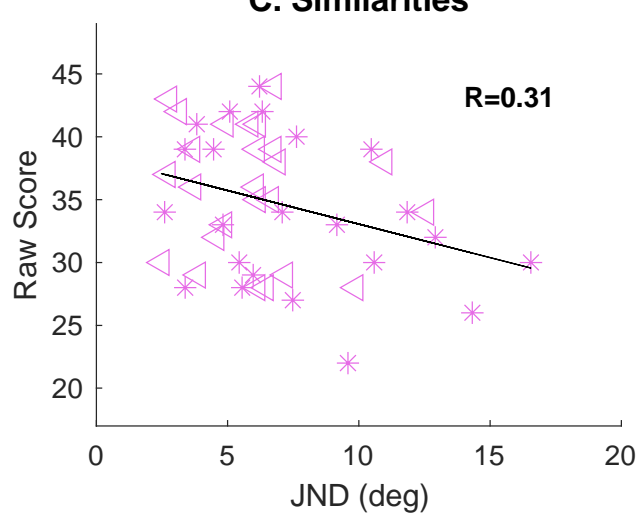

D. Matrix Reasoning

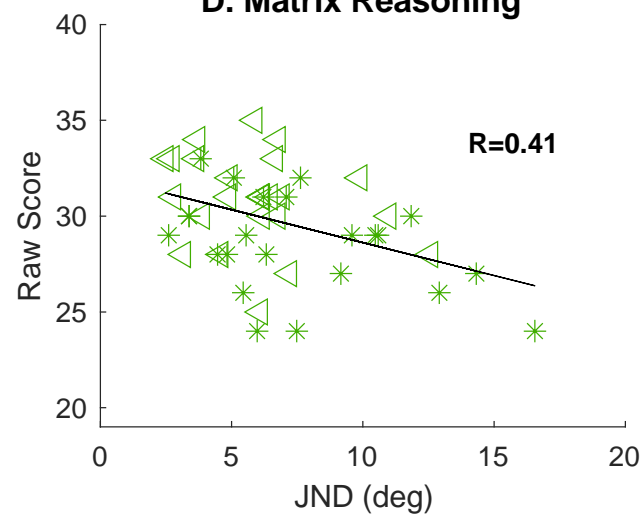

\section{Figure 4}

Individual raw scores from the four subtests correlated against JND in degrees of orientation $(N=48)$. Verbal subtests illustrated in pink, non-verbal subtests in green. Triangles indicate males, stars indicate females. A. Vocabulary raw scores show a good negative correlation with orientation discrimination abilities $\left(R=0.39, R^{2}=0.15, p=0.006\right)$. B. Block Design raw scores 
show a weaker correlation with orientation discrimination abilities $\left(R=0.32, R^{2}=0.1\right.$, p=0.025). C. Similarities raw scores show a negative correlation with orientation discrimination abilities, similar to $B\left(R=0.31, R^{2}=0.1, p=0.032\right)$. D. Matrix Reasoning raw scores show the strongest negative correlation with $\operatorname{JND}\left(R=0.41, R^{2}=0.17, p=0.004\right)$.

In order to explore the relation between Block Design and orientation discrimination abilities, we correlated scores on this WAIS subtest to JND. We found a negative correlation $(\mathrm{R}=0.32$, $\mathrm{R}^{2}=0.1, \mathrm{p}=0.025$ ) between Block Design individual raw scores and JND (Figure 4B). Figure 4D shows that individual scores from the Matrix Reasoning subtest have the strongest negative correlation among the four subtests with JND $\left(\mathrm{R}=0.41, \mathrm{R}^{2}=0.17, \mathrm{p}=0.004\right)$ suggesting that participants who have higher scores in the Matrix Reasoning have lower JND values, thus more accurate orientation discrimination skills.

Next, we computed correlations among the four subtests (Figure 5). The strongest correlations are observed between Similarities and the other three subtests. The two verbal subtests, Similarities and Vocabulary show the strongest positive correlation (Figure 5B, $\mathrm{R}=0.634, \mathrm{p}<0.001$ ). Block Design (Figure 5D, $\mathrm{R}=0.447, \mathrm{p}=0.001$ ) and Matrix Reasoning (Figure 5F, $\mathrm{R}=0.442, \mathrm{p}=0.002$ ) also show strong correlations with Similarities. The weakest correlation is observed between individual raw scores of the Vocabulary and Matrix Reasoning subtests (Figure $5 \mathrm{C}, \mathrm{R}=0.316, \mathrm{p}=0.029$ ), suggesting that the strong relationship of the two with orientation discrimination abilities observed in Figure 4, could be driven by distinct factors. Scores in the two non-verbal subtests, Matrix Reasoning and Block Design were also positively correlated (Figure 5E, R=0.407, p=0.004). Finally, scores in the Block Design were positively correlated with Vocabulary (Figure 5A, $\mathrm{R}=0.407, \mathrm{p}=0.004$ ). 

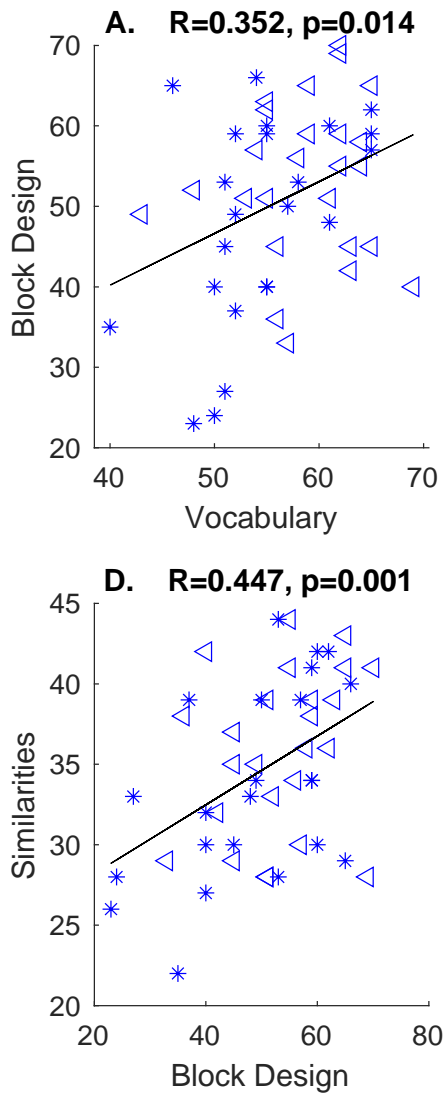

B. $R=0.634, p=0$

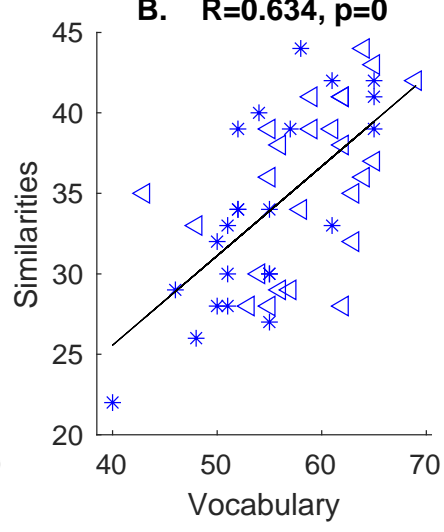

E. $R=0.407, p=0.004$

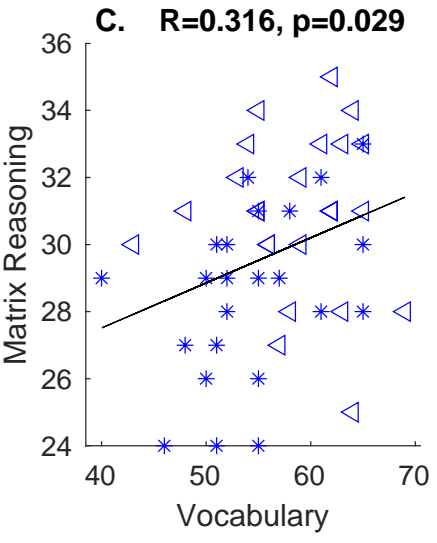

F. $R=0.442, p=0.002$


\section{Figure 5}

Individual raw scores from the four subtests correlated against each other $(N=48)$. Triangles indicate males, stars indicate females. All subtests are correlated with one another. A. Block design vs. Vocabulary $(R=0.407, p=0.004)$, B. Similarities vs. Vocabulary $(R=0.634$, $p$ <0.001). C. Matrix Reasoning vs. Vocabulary $R=0.316, p=0.029$ D. Similarities vs. Block Design ( $R=0.447, p=0.001)$ E. Matrix Reasoning vs. Block Design $(R=0.407, p=0.004), F$. Matrix Reasoning vs. Similarities $(R=0.442, p=0.002)$.

Given the significant correlations observed between individual raw scores of the four subtests (Figure 5), we calculated partial correlations for each subtest with JND, while controlling for the effect of one other subtest. It is important to note here that we do not perform a multiple regression which would be the preferred statistical test, as the assumption of independent variables is violated. Table 1 shows that the Matrix Reasoning is the only subtest which preserves a strong negative correlation with orientation discrimination abilities, after controlling for the effects of the other three subtests (Vocabulary: $\mathrm{R}=-0.33, \mathrm{p}=0.02$, Block Design: $\mathrm{R}=-0.32, \mathrm{p}=0.03$, Similarities: $\mathrm{R}=-0.32, \mathrm{p}=0.03$ ). Vocabulary maintains a strong 
negative correlation with JND when controlling for Block Design ( $R=-0.31, p=0.03)$ and Matrix Reasoning $(\mathrm{R}=-0.30, \mathrm{p}=0.04)$, but not when controlling for the verbal subtest Similarities $(\mathrm{R}=-0.27, \mathrm{p}=0.07)$. Block Design and Similarities show no significant correlation with JND after controlling for the effects of the other subtests.

Correlated subtest

Controlling for

\begin{tabular}{lcccc} 
& Vocabulary & Block Design & Similarities & Matrix Reasoning \\
\hline Vocabulary & -- & $\mathbf{- 0 . 3 1}$ & -0.27 & $\mathbf{- 0 . 3 0}$ \\
$(0.39)$ & $(p=0.03)$ & $(p=0.07)$ & $(\boldsymbol{p}=\mathbf{0 . 0 4})$ \\
\hline Block design & -0.19 & -- & -0.22 & -0.19 \\
$(0.32)$ & $(p=0.21)$ & & $(p=0.14)$ & $(p=0.21)$ \\
\hline Similarities & -0.09 & -0.20 & -- & -0.16 \\
$(0.31)$ & $(p=0.56)$ & $(p=0.19)$ & & $(p=0.29)$ \\
\hline Matrix Reasoning & $\mathbf{- 0 . 3 3}$ & $\mathbf{- 0 . 3 2}$ & $\mathbf{- 0 . 3 2}$ & -- \\
$(0.41)$ & $(\boldsymbol{p}=\mathbf{0 . 0 2})$ & $(\boldsymbol{p}=\mathbf{0 . 0 3})$ & $(\boldsymbol{p}=\mathbf{0 . 0 3})$ &
\end{tabular}

Table 1

Partial correlations of individual raw values from each subtest with JND, while controlling for one of the other three subtests. Vocabulary has no significant correlation with JND when controlling for Similarities but preserves a significant negative correlation when controlling for Block design or Matrix Reasoning. Block design (2 ${ }^{\text {nd }}$ row) and Similarities ( $3^{\text {rd }}$ row) subtests appear to have no significant correlation with JND when controlling for the other subtests. Matrix reasoning ( $4^{\text {th }}$ row) maintains a significant negative correlation with JND, when controlling for the effects of any other subtest.

Taken together, our results show that Matrix Reasoning is the strongest predictor of orientation discrimination abilities as measured by JND, even after controlling for the effects of the other three subtests of WASI-II. To our surprise, Vocabulary also appears to have a strong negative correlation with JND, after controlling for the effects of the two non-verbal subtests (Block Design, Matrix Reasoning). 


\section{Discussion}

The aim of this study was to investigate whether basic sensory information acts as a building block for much more complex cognitive processes. The novelty lies in the visual task we have used which evaluates one of the simplest abilities of any visual system: orientation discrimination. The significant correlations we found between overall intelligence scores and orientation discrimination abilities, as measured by the Just Noticeable Difference (JND), documented that participants with higher IQ scores have more accurate orientation discrimination skills. Non-verbal skills, measured with the Matrix Reasoning and Block Design subtests and verbal skills assessed with the Vocabulary and Similarities subtests, showed strong correlations with JND as well. Each standardised WASI subtest was also individually correlated with JND, with Matrix Reasoning exhibiting the highest correlation. Given that such correlations could be driven by allocation of attentional resources or Working Memory (WM) capacity, we calculated partial correlations to reveal truly independent correlations with orientation discrimination skills. We report significant partial correlations with Matrix Reasoning (accounting for any of the three other subtests) and Vocabulary (accounting for Block Design and Matrix Reasoning).

Simple correlations of Similarities and Block Design with orientation discrimination abilities may potentially be driven by other, more general intelligence factors. Given that participants were asked to compare two Gabor gratings presented within only a 2 second window, the task administered to the participants could be considered a short-term memory task. Thus, short-term memory may be at the heart of these associations. Spearman (1904) first observed the phenomenon 'positive manifold', where many kinds of cognitive tests (i.e. arithmetic and vocabulary) were all positively correlated with each other. Similarly, Jensen (2000) suggested that by examining various results from general intelligence tests (from simple sensory discrimination to reaction times of highly complex problem solving), the degree of correlation between them can be boiled down to physiological aspects such as brain size, brain nerve conduction velocity and brain glucose metabolic rate. Indeed, Colom and colleagues (2006) found that participants scoring higher in intelligence tests had greater grey matter volume in all brain lobes, suggesting that perhaps g relates to distributed networks across the brain. 
Further into this, Kovacs and Conway (2016), introduced Process Overlap Theory, a unified account of the general factor of intelligence " $\mathrm{g}$ ", which states that during cognitive tests, executive processes are tapped into in an overlapping manner. According to this, there are distinct within-individual processes tapped by different test items which might belong to different cognitive domains. Such processes could give rise to apparent correlations between our subtests and orientation discrimination abilities, while in fact the true correlation is driven by central executive processes and the ability to allocate cognitive resources in order to complete a specific task. Therefore, the reported significant partial correlations of Matrix Reasoning and Vocabulary subtests with orientation discrimination abilities suggest that each subtest maintains an independent relationship with orientation discrimination abilities which is not based simply on allocation of attention or WM capacity. This idea of a central integrative system of coordinating between processes (Baddeley \& Hitch, 1974), describes a mechanism through which information could be maintained in a readily accessible state but could also engage in concurrent processing, while still having access to long-term memories. Later, Baddeley (2001) developed a more nuanced model of memory and clarified that communication between each subsystem is based on the idea that that such transformations critically rely on the central executive.

Orientation discrimination abilities are thought to be defined by the primary visual cortex (V1) which is traditionally thought to operate in a purely sensory manner by receiving direct retinal input from the eyes and constructing an accurate retinotopic map of the visual world. Rademaker et al. (2019) suggested that in reality V1 may potentially possess coexisting representations of both sensory and mnemonic information, running via a 'local comparison circuit'. Separable bottom-up and top-down inputs could theoretically support the coexistence of multiple simultaneous representations. Population-level response patterns in early visual cortex were found to represent the contents of working memory alongside new sensory inputs. In a second experiment, Rademaker et al. (2019) showed that when participants got distracted, both disruptions of mnemonic information in early visual cortex and decrements in behavioural recall were observed. Therefore, it can be inferred that information already present in working memory (from early retinotopic cortex) is maintained even after new sensory inputs are processed into visual working memory. However, salient and distracting information can negatively impact recall, suggesting that early visual areas actively participate in both sensory and mnemonic processing. In support of this notion, Vredeveldt, Hitch and Baddeley (2011) showed that eye closure reduces cognitive load and 
aids memory recall by exposing 80 participants to a violent video clip and later to different types of distraction during the witness interview (blank screen- control, eyes closed, visual distraction, auditory distraction). Recall was significantly better when distraction was minimal, providing evidence that eye closure reduces input to $\mathrm{V} 1$ thus reducing cognitive load.

Our results appear to agree with the broader literature regarding sensory discrimination and intelligence. The good correlation with Matrix Reasoning comes as no surprise as the subtest requires excellent visual skills including classification, spatial abilities, knowledge of part-whole relationships and perceptual organization. These results agree with Melnick et al.'s (2013) conclusions discussed in the introduction, that accuracy and speed of processing of low-level visual properties may predict higher intellect in individuals. Their results suggest that visual suppression mechanisms play a crucial role in perception by allowing our perceptual systems to efficiently process vast amounts of sensory input (Carandini \& Heeger, 2012), in addition to an analogous role in intelligent cognition by contributing to improved neural efficiency (Burgess et al., 2011). Our results go a step further to show that orientation discrimination, probably the most basic of all visual skills, can be indicative of overall intelligence skills.

Neural suppression efficiency could provide a mechanistic explanation for the SI - IQ link, through multiple inhibitory processes which have been found to strongly predict IQ scores (Friedman \& Miyake, 2004), such as attentional and working-memory control (Engle et al., 1999). Cook et al. (2016) reported that higher levels of cortical gamma-aminobutyric acid (GABA) in the human primary visual cortex were associated with better performance in the Matrix Reasoning IQ subtest $(r=0.83, p=0.0054)$. Further, Edden and colleagues (2009) added that neuronal inhibition is mediated by GABAergic interneurons, possibly influencing performance in orientation judgement tasks. Thus, GABA constitutes a plausible candidate neural substrate for Melnick et al.'s proposal that the link between SI and IQ in the suppression of irrelevant information.

The correlations between raw scores of the Vocabulary subtest and JND could therefore be attributed to information suppression abilities: participants who required fewer degrees of difference to realise that two successive Gabor patches differed in orientation, can probably 'shed' unnecessary information more effectively in general. The importance of vision in language acquisition through reading and writing in neurotypical and sighted humans could also explain this relationship. Bedny et al., (2011) showed that cortical regions 
in the occipital lobe of blind human adults typically specialised in visual processing in sighted adults, appear to be taking on language processing as a result of early experience. Such cortical reorganisation has been widely reported in the literature and it is believed to facilitate the efficient processing of sensory input by available and capable brain areas (Ajina et al., 2015), in this case the occipital cortex of blind individuals. This would suggest that the occipital cortex has the capacity to process language at a supra-modal level.

Our results provide evidence that low-level visual abilities and high-level cognitive processes are more tightly interwoven than previously thought. This is of great importance, as basic vision research is often overlooked by researchers who focus on higher-level and often multisensory human abilities, assuming that basic visual abilities cannot be directly associated with more advanced thinking. 


\section{Author Contributions}

K.M. designed the study and analysed the data. K.M., N.L., E.G. performed the experiments. All authors discussed results, wrote and reviewed the main manuscript text.

\section{Acknowledgments}

This research was supported by the European Union's Horizon 2020 research and innovation programme under the Marie Skłodowska-Curie grant agreement 'Peripheality' no. 797603 (K.M.).

\section{References (max 40)}

Acton, G. S., \& Schroeder, D. H. (2001). Sensory discrimination as related to general intelligence. Intelligence, 29(3), 263-271. https://doi.org/10.1016/S0160-2896(01)00066-6

Ajina, S., Kennard, C., Rees, G., \& Bridge, H. (2015). Motion area V5/MT+ response to global motion in the absence of V1 resembles early visual cortex. Brain, 138(1), 164-178.

Arranz-Paraíso, S., \& Serrano-Pedraza, I. (2018). Testing the link between visual suppression and intelligence. PLOS ONE, 13(7), e0200151. https://doi.org/10.1371/journal.pone.0200151

Baddeley, A. D. (2001). Is working memory still working? American Psychologist, 56(11), 851864. https://doi.org/10.1037/0003-066X.56.11.851

Baddeley, A. D., \& Hitch, G. (1974). Working Memory. In Psychology of Learning and Motivation (Vol. 8, pp. 47-89). Elsevier. https://doi.org/10.1016/S0079-7421(08)60452-1

Bedny, M., Pascual-Leone, A., Dodell-Feder, D., Fedorenko, E., \& Saxe, R. (2011). Language processing in the occipital cortex of congenitally blind adults. Proceedings of the National Academy of Sciences, 108(11), 4429-4434. https://doi.org/10.1073/pnas.1014818108

Brainard, D. H. (1997). The Psychophysics Toolbox. Spatial Vision, 10(4), 433-436. $\underline{\text { https://doi.org/10.1163/156856897X00357 }}$

Burgess, G. C., Gray, J. R., Conway, A. R. A., \& Braver, T. S. (2011). Neural mechanisms of interference control underlie the relationship between fluid intelligence and working memory 
span. Journal of Experimental Psychology: General, 140(4), 674-692. https://doi.org/10.1037/a0024695

Carandini, M., \& Heeger, D. J. (2012). Normalization as a canonical neural computation. Nature Reviews Neuroscience, 13(1), 51-62. https://doi.org/10.1038/nrn3136

Colom, R., Jung, R. E., \& Haier, R. J. (2006). Distributed brain sites for the g-factor of intelligence. NeuroImage, 31(3), 1359-1365.

https://doi.org/10.1016/j.neuroimage.2006.01.006

Cook, E., Hammett, S. T., \& Larsson, J. (2016). GABA predicts visual intelligence. Neuroscience Letters, 632, 50-54. https://doi.org/10.1016/j.neulet.2016.07.053

Deary, I. J. (1994). Sensory discrimination and intelligence: postmortem or resurrection?. The American journal of psychology, 95-115.

Edden, R. A. E., Muthukumaraswamy, S. D., Freeman, T. C. A., \& Singh, K. D. (2009). Orientation Discrimination Performance Is Predicted by GABA Concentration and Gamma Oscillation Frequency in Human Primary Visual Cortex. Journal of Neuroscience, 29(50), 15721-15726. https://doi.org/10.1523/JNEUROSCI.4426-09.2009

Engle, R. W., Tuholski, S. W., Laughlin, J. E., \& Conway, A. R. A. (1999). Working memory, short-term memory, and general fluid intelligence: A latent-variable approach. Journal of Experimental Psychology: General, 128(3), 309-331. https://doi.org/10.1037/0096$\underline{3445.128 .3 .309}$

Friedman, N. P., \& Miyake, A. (2004). The Relations Among Inhibition and Interference Control Functions: A Latent-Variable Analysis. Journal of Experimental Psychology: General, 133(1), 101-135. https://doi.org/10.1037/0096-3445.133.1.101

Jensen, A. R. (2008). The $g$ Factor: Psychometrics and Biology. In G. R. Bock, J. A. Goode, \& K. Webb (Eds.), Novartis Foundation Symposia (pp. 37-57). John Wiley \& Sons, Ltd. https://doi.org/10.1002/0470870850.ch3 
Kovacs, K., \& Conway, A. R. A. (2016). Process Overlap Theory: A Unified Account of the General Factor of Intelligence. Psychological Inquiry, 27(3), 151-177. $\underline{\text { https://doi.org/10.1080/1047840X.2016.1153946 }}$

Melnick, M. D., Harrison, B. R., Park, S., Bennetto, L., \& Tadin, D. (2013). A Strong Interactive Link between Sensory Discriminations and Intelligence. Current Biology, 23(11), 10131017. https://doi.org/10.1016/j.cub.2013.04.053

Rademaker, R. L., Chunharas, C., \& Serences, J. T. (2019). Coexisting representations of sensory and mnemonic information in human visual cortex. Nature Neuroscience, 22(8), 1336-1344. https://doi.org/10.1038/s41593-019-0428-X

Spanoudis, G., \& Tourva, A. (2012). Greek-Cypriot Wechsler Abbreviated Scale of Intelligence (WASI). Nicosia: University of Cyprus

Spearman, C. (1904). "General intelligence" objectively determined and measured. American Journal of Psychology, 15, 201-293.

Tadin, D. (2015). Suppressive mechanisms in visual motion processing: From perception to intelligence. Vision Research, 115, 58-70. https://doi.org/10.1016/j.visres.2015.08.005

Tadin, D., Lappin, J. S., Gilroy, L. A., \& Blake, R. (2003). Perceptual consequences of centresurround antagonism in visual motion processing. Nature, 424(6946), 312-315. https://doi.org/10.1038/nature01800

Vredeveldt, A., Hitch, G. J., \& Baddeley, A. D. (2011). Eyeclosure helps memory by reducing cognitive load and enhancing visualisation. Memory \& Cognition, 39(7), 1253-1263. https://doi.org/10.3758/s13421-011-0098-8 


\section{Appendix I}

\section{A) Vocabulary}

This subscale includes 42 objects: 4 images and 38 words. For images, the participant needs to name what they see. For words, the participant gives their meaning orally and answers are recorded word for word. For participants aged between 9-89 the assessment starts from object 9 , including only words. If the participant scores full marks ( 2 points) on both objects 9 and 10 then full marks are given for objects 1-8. If the participant scores 0 or 1 on object 9 or 10 , then we continue with objects 5-8 in a reverse order, until the participant scores 2 points to 2 continuous objects, including object 9 if it has full marks. When this part is complete, we give full marks to objects 5-8 which were not administered. Then we continue with the subscale until the interruption criteria or until the end. If the participant scores 0 or 1 to object 5 or 6 , then we administer objects 1-4. After this, we continue with the rest of the objects in this subscale. We interrupt the vocabulary subscale after 5 continuous failed attempts (' 0 ' scores), otherwise for ages 17-89, we continue until the last object of the subscale. There are no strict time limits for the vocabulary, but typically participants need less than 30 seconds to provide a response for each object. For objects 1-4, we give 0 or 1 points and for objects 5-42 we give 0,1 or 2 points according to the answer given by the participant. Highest raw score for ages $17-89$ is 80 .

\section{B) Block Design}

In this subscale, the participant is required to reproduce two-dimensional bicolour designs printed on paper using wooden bi-coloured cubes within the specified timeframe. There are 13 designs with an increasing level of difficulty, starting with the simple ones which should be constructed with only 4 cubes and progressing to the most difficult ones with 9 cubes. Every cube has 2 white sides, 2 red sides and 2 bicolor sides (both red and white). For ages 9-89 we begin with object 3 . If the participant scores full marks ( 2 points) on both objects 3 and 4 , we also give full marks on objects 1 and 2 . If they score 0 or 1 points on object 3 or 4 , then we administer objects 1 and 2 in reverse order until full marks are received in 2 continuous objects, (including object 3 if it has full marks). When this part is completed, we give full marks to the rest of the objects which were not administered. Then we continue until the end of the subscale. If the participant scores 0 points to 3 continuous objects, we stop testing for this subscale. For objects 1-4 participants have a second chance, unlike objects 5-13. It is necessary to show all different sides of the cubes before the participant begins the task. We have to strictly follow the time limits given for each object, especially on objects 5-13 because it's important for the 
allocation of extra points. Participants are allowed to make changes if they find out they have made a mistake within the allocated time frame. For objects 5-9, the time limit is 1 minute and for objects 10-13, 2 minutes. The marking is based on the correctness of the design and the time taken to be complete it. A design can be considered erroneous because of wrong structure, a rotation of 30 degrees and more, or transcendence of the time limit. For objects 1-4, we give full marks ( 2 points) if the design is reproduced in the first attempt. If there is a second chance, then we give 1 point. For ages 9-89 who scored full marks on both objects 3 and 4, we also give full marks for objects 1 and 2. For objects 5-13, the marks are given according to the time taken to complete it. Highest raw score for all ages is 71 .

\section{C) Similarities}

In this subscale, there are 26 objects. For objects 1-4, on paper there are 2 rows with images 3 images in the upper row and 4 at the bottom. The participant has to recognize the image of the bottom row which is similar to the images in the upper row. For objects 5-26, we have to read out loud 2 words which represent common meanings. The participant is called to formulate how these 2 words are similar. For ages $12-89$ we start with object 7 . If the participant scores full marks ( 2 points) on both objects 7 and 8 , then we also give full marks for objects 16. If the participant scores 0 or 1 points to object 7 or 8 then we administer objects 5 and 6 in a reverse order until they score full marks to 2 continuous objects (including object 7 if it has full marks). When this part is completed, full marks are given to all previous objects which were not administered. Then, we continue with the subscale until the interruption criteria or until the end. If the participant scores 0 or 1 to object 5 or 6 , then we administer objects 1-4. Then, we continue with the subscale until the interruption criteria or until the ending point. We interrupt the subscale after 4 continuous ' 0 ' scores, otherwise for ages 12-89 we continue until the last object of the subscale. If participants do not provide an answer within 30 seconds, we mark the object as erroneous and continue with the next one. Objects 1-4 are ranked with 0 or 1 points. Objects 5-26 are ranked with 0,1 or 2 points. Full marks are given for previous objects which are not administered. An answer scores 2 points when it constitutes a general category which matches perfectly both words. An answer is awarded 1 point when it reflects a general category, but it is not the most accurate one which describes what is common between the words. Lastly, an answer is ranked with 0 points when it does not describe what is common between the two words. If the participant gives an answer which should be awarded with 2 or 1 points but also gives an erroneous answer, no points are awarded. However, if the participant 
gives multiple answers which differ a lot in quality, but none of them is erroneous, then we record and score the best one. Highest raw score for ages 12-89 is 48 .

\section{D) Matrix Reasoning}

Four types of non-verbal reasoning capacities constitute this subscale: pattern completion, categorization, proportions and serial logic. The participant has to find the missing piece of the pattern, choosing between 5 options. For ages 12-44, it is necessary to first show examples A and B and then start from object 7. If the participant scores full marks (1 point) for both objects 7 and 8, we also give full marks for objects 1-6. If the participant scores 0 points to object 7 or 8 then we administer objects 1-6 in a reverse order until they score full marks to 2 continuous objects (including object 7 if it has full marks). When this part is complete, we give full marks to the objects which were not administered. Then, we continue with the subscale until the interruption criteria or until the end. We interrupt the subscale after 4 continuous ' 0 ' scores, or 4 ' 0 ' scores to 5 continuous objects, otherwise for ages 12-44, we continue until the last object of the subscale. There is no strict time limit but typically participants need 30 seconds for each object. If a participant needs more than 30 seconds, we continue with the next object and count the previous one as a failure. Highest raw score for ages 12-44 is 35 . 